\title{
Why Feature Dependencies Challenge the Requirements Engineering of Automotive Systems: An Empirical Study
}

\author{
Andreas Vogelsang \\ Institut für Informatik \\ Technische Universität München \\ Boltzmannstr. 3, 85748 Garching, Germany \\ vogelsan@in.tum.de
}

\author{
Steffen Fuhrmann \\ BMW Group \\ Driving Dynamics \\ Dimensioning Functions Driving Dynamics and Driver Assistance \\ Knorrstr. 147, 80788 München, Germany \\ steffen.fuhrmann@bmw.de
}

\begin{abstract}
Functional dependencies and feature interactions in automotive software systems are a major source of erroneous and deficient behavior. To overcome these problems, many approaches exist that focus on modeling these functional dependencies in early stages of system design. However, there are only few empirical studies that report on the extent of such dependencies in industrial software systems and how they are considered in an industrial development context. In this paper, we analyze the functional architecture of a real automotive software system with the aim to assess the extent, awareness and importance of interactions between features of a future vehicle. Our results show that within the functional architecture at least $85 \%$ of the analyzed vehicle features depend on each other. They furthermore show that the developers are not aware of a large number of these dependencies when they are modeled solely on an architectural level. Therefore, the developers mention the need for a more precise specification of feature interactions, e.g., for the execution of comprehensive impact analyses. These results challenge the current development methods and emphasize the need for an extensive modeling of features and their dependencies in requirements engineering.

Index Terms-Functional specifications, feature interaction,
\end{abstract} model-based development, automotive, empirical studies

\section{INTRODUCTION}

The behavior of software-intensive embedded systems is characterized by its features and functions. Many modelbased specification techniques for software-intensive systems utilize this notion of a system feature in order to structure a specification (e.g., [1], [2], [3]). Although the notions in the different approaches differ slightly and sometimes synonyms such as "system function", "feature", or "user function" are used, the approaches agree on structuring a specification into subparts that contain extracts of the functionality as it is perceived by the user or any other environmental system. We will call these parts system features or vehicle features in the remainder of the paper.

Dependencies and interactions between these features are a major challenge in the industrial development of software-

This work was partly funded by the German Federal Ministry of Education and Research (BMBF), grant "FoMoStA, 01IS12028". intensive systems [4]. They increase the complexity of the system and frequently entail unwanted and deficient system behavior [5]. Nevertheless, feature interactions are rarely considered in specifications of industrial systems. This leads to increased efforts in late development phases like integration or system test when these errors typically are revealed [5]. We use the terms functional dependencies and feature interaction synonymously in this paper.

These dependencies play an important role especially in the development of multifunctional systems such as automotive software systems [6], [7]). However, dependencies induced by multifunctionality are a major challenge even for the development of embedded systems in general.

In order to handle functional dependencies, many approaches exist, which model such dependencies in early phases of the development process, i.e., in the specification or the system design (e.g., [1], [2], [8]).

\section{A. Problem Statement}

Existing approaches towards modeling of functional dependencies have been validated in specific examples and applications. However, there is little empirical data on the extent and distribution of such dependencies in industrial software systems and their consideration in an industrial development context. It is therefore not possible to thoroughly assess the impact and influence of functional dependencies and feature interactions on the development of modern software systems. A comprehensive understanding of the awareness and importance of such dependencies in system development is further mandatory to evaluate existing modeling approaches.

In a previous study concerning the extent and characteristics of functional dependencies in automotive software systems, we observed a large number and a complex nature of such dependencies [9]. For the further investigation of functional dependencies and the validation of our earlier results, larger systems with a more networked architecture should be evaluated. Furthermore, the quantitative analysis of our former study neglects a qualitative assessment of the feature interactions. A 
qualitative study is thus necessary to discover the impact on development methods and processes.

\section{B. Research Objective}

The purpose of our two-phase, sequential mixed methods study is to obtain quantitative results from a sample and then follow up with a few individuals to explore those results in depth. In the first phase, a quantitative research question will address the extent of feature dependencies in a modern automotive software system. We aim at the identification of valuebased dependencies between vehicle features. We therefore extract data flow dependencies from the functional architecture of a software system and assess them concerning their value on a vehicle level. In the second phase, qualitative interviews will be used to probe the awareness of the found dependencies and discover the importance for automotive software developers.

\section{Contribution}

We analyzed the functional architecture of a real automotive software system in order to contribute

- data on the amount and distribution of functional dependencies between vehicle features,

- an evaluation of how dependencies are considered throughout the automotive development process.

The results of our study motivate the use of more extensive modeling techniques for features and their dependencies.

\section{Context}

The study focuses on automotive software systems and was executed at the BMW Group, a German manufacturer of premium automobiles and motorcycles. We analyzed the functional architecture of driving dynamics and driver assistance systems that will be implemented in a future sports utility vehicle $(S U V)$. The functional architecture consists of vehicle features, which are grouped into certain feature groups building a hierarchy of vehicle features. The atomic vehicle features in this hierarchy are realized by a network of logical components that we refer to as leaf functions.

In our context, vehicle features and leaf functions build the central elements for the specification of functional requirements. Subject to their level of detail, requirements must apply either to the definition of a vehicle feature or a leaf function. Dependencies within the functional architecture therefore entail dependencies between functional requirements.

Within the driving dynamics and driver assistance domain, an in-house developed database supports the design and implementation of the functional architecture. Based on this data backbone, a model-based development approach ensures the realization of the functional architecture by program code, sensors and actuators. For the analyses in our study, we used a specific dataset that we extracted from the described data backbone.

\section{RELATED WORK}

Except from our previous study [9] and to the best of our knowledge, there is no comparable work on empirical data or analyses of realistic automotive or embedded systems with the focus on dependencies between system features. However, there is a lot of work on approaches that try to model or specify such dependencies.

Functional dependencies and feature interactions have been extensively investigated in the telecommunication domain [10]. Jackson and Zave [1] introduced Distributed Feature Composition $(D F C)$ as a modular, service-oriented architecture for applications in the telecommunication domain. DFC relies on the notion that a user service request can be composed of a set of smaller features, which are arranged in a pipesand-filters architectural style. This architecture is especially designed for modeling interactions between different features.

Classical approaches like UML use cases, activities or sequences [11] specify system features more or less in isolation. Dependencies between system features are neglected. This makes it hard to reason about functionality that arises from the interplay of multiple system features.

A well-known specification technique for requirements is the software cost reduction (SCR) method [8]. In SCR, requirements are specified by a set of specification tables. The developers of SCR also noticed that understanding the relationship between different parts of a specification can be difficult, especially for large specifications [12]. Therefore, a Dependency Graph Browser in their tool displays the dependencies among the variables in a given specification.

\section{STUdy DESIGN}

In this section, we formulate the research questions, describe the study object as well as the data collection and analysis procedures. We conclude with a description of validity procedures.

\section{A. Research Questions}

The study examines the amount of functional dependencies in automotive software systems and how dependencies are handled in the development process. We assess the awareness and importance of functional dependencies to justify the application of feature modeling approaches. We structured our study with the help of three research questions.

RQ 1: What is the overall extent and distribution of dependencies between vehicle features?

The relevance of functional dependencies can be motivated inter alia by an analysis of the overall number of interactions. We define a dependency between vehicle features as an influence on the behavior of a vehicle feature by the state or data of another vehicle feature.

RQ 2: To what extent are developers aware of functional dependencies?

Developers of automotive systems are not necessarily aware of existing dependencies and interactions. We want to identify existing feature dependencies that are unknown to developers as well as known dependencies that are not represented within the functional architecture and assess them with regard to their plausibility. 


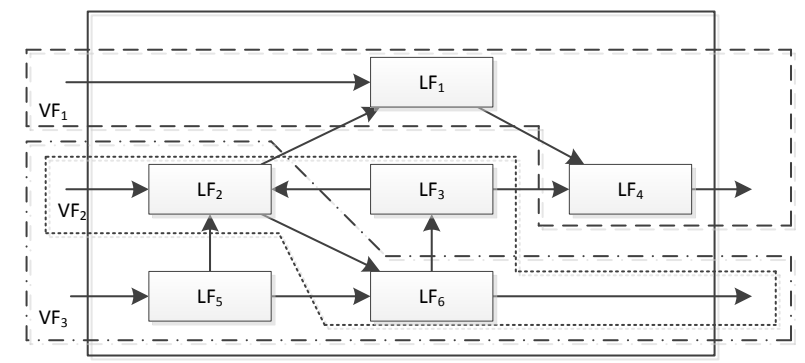

Fig. 1. The leaf functions (rectangles) are connected by data channels (black arrows) and form a functional architecture of the system (outer rectangle). Vehicle features crosscut this architecture by the set of leaf functions that contribute to their realization (dashed forms).

RQ 3: How important is a comprehensive understanding of functional dependencies and feature interactions?

We have to investigate existing feature interactions concerning their importance for the development process and design decisions in order to reason an extensive modeling of feature interactions.

\section{B. Study Object}

In our study we analyzed an automotive software system of a future vehicle and especially its functional architecture. Within the functional architecture we focused on the driving dynamics and driver assistance domain. The system comprises 94 vehicle features and a total of 325 leaf functions. Leaf functions may be used for the realization of more than one vehicle feature.

Leaf functions describe the realization/implementation of a vehicle feature in a purely logical fashion, i.e. without any information about the hardware the system runs on. A network of leaf functions describes the steps that are necessary to transform the input data into the desired output data. An example for a system that consists of 3 vehicle features, which are realized by a network of 6 leaf functions is illustrated in Fig. 1. The leaf functions are afterwards assigned to specific software components, which execute the behavior of the leaf functions. As a final step, these software components are deployed to a set of electronic computing units.

The relation between a vehicle feature and a leaf function in the context of this study is the following: A vehicle feature is realized by a set of leaf functions that are arranged in a data-flow network. A leaf function can contribute to the realization of a set of vehicle features. Thus, there is an $n: m$ relation between vehicle features and leaf functions. The set of all leaf functions and their connections form the functional architecture of the system. The vehicle features crosscut this architecture by the set of leaf functions that contribute to their realization (see Fig. 1).

\section{Data Collection Procedures}

For the reliable acquisition of data, we need a precise definition of what we consider as a dependency between vehicle features. Our initial informal definition states that a

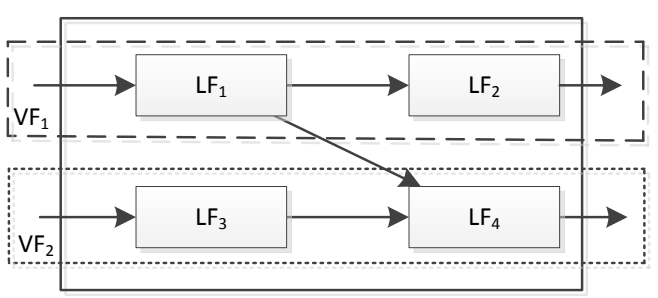

Fig. 2. The vehicle feature $V F_{2}$ depends on the vehicle feature $V F_{1}$, since the leaf function $L F_{1}$ (part of $V F_{1}$ ) sends values to the leaf function $L F_{4}$ (part of $V F_{2}$ ). Thus, the behavior of $V F_{2}$ depends on data of $V F_{1}$.

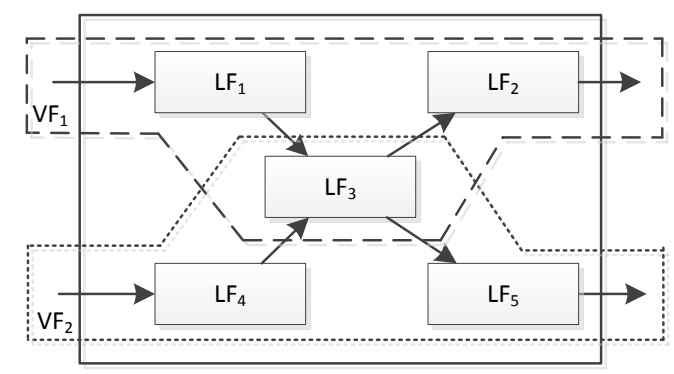

Fig. 3. Vehicle features $V F_{1}$ and $V F_{2}$ share the leaf function $L F_{3}$. This might indicate a dependency between the vehicle features. However, this cannot be verified without further knowledge about the behavior of $L F_{3}$.

vehicle feature $V F_{1}$ depends on another vehicle feature $V F_{2}$ if its behavior is influenced not only by its primary inputs but also by the state or data of $V F_{2}$. Therefore, the vehicle features have to communicate with each other. In our study, we distinguish between two different ways of communication between vehicle features.

1) A leaf function that is part of one vehicle feature has a communication channel to a leaf function that is part of another vehicle feature (see Fig. 2).

2) A leaf function is related to two or more vehicle features, i.e., two or more vehicle features share a leaf function (see Fig. 3).

However, a real dependency between two vehicle features cannot be derived definitely from the shared use of a leaf function. In that case, further knowledge about the concrete behavior of the leaf function is needed in order to identify the possible dependency. In the example of Fig. 3 , the influence of the data transmitted over the channel $L F_{1} \rightarrow L F_{3}$ on the data transmitted over the channel $L F_{3} \rightarrow L F_{5}$ can only be assessed with further knowledge about the behavior of $L F_{3}$.

As we had no information about the precise behavior of leaf functions in the context of our study, we focused on dependencies of type 1, where a leaf function of one vehicle feature has a communication channel to a leaf function of another vehicle feature.

Based on this definition of a dependency between vehicle features, we can extract a vehicle feature graph from the 


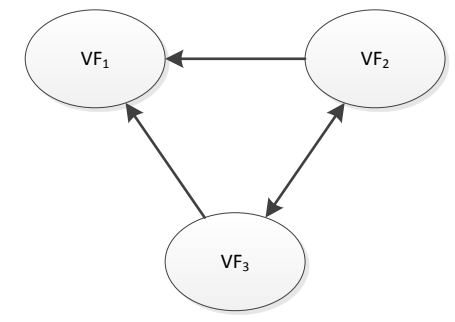

Fig. 4. The vehicle feature graph extracted from the functional architecture of Fig. 1

functional architecture, where each node is a vehicle feature and a directed edge indicates a dependency between two vehicle features. The resulting vehicle feature graph for the example of Fig. 1 is illustrated in Fig. 4.

In our study, we extracted the vehicle feature graph by means of a simple tool, written in Java. The tool parses an exported data set from the company's data backbone containing a list of features associated with a set of leaf functions. The tool transforms the data into a graph structure, extracts the feature dependencies according to the definition given in this section, and finally outputs a .csv file with the found dependencies. The extraction was performed fully automated and the complexity of the algorithm is quadratic in the number of vehicle features and leaf functions. For the observed system, the extraction took around 3 seconds on a standard laptop.

The second part of our study is based on four interviews with feature experts from the BMW Group, who are involved in the design of the functional architecture.

For RQ 2, we confronted the experts with a sample of feature dependencies from their area of responsibility found by our analysis. We let the experts classify these dependencies into the following categories:

- plausible/implausible: A dependency is considered as plausible if the expert finds a functional or physical explanation for this dependency. If the expert has no functional or physical explanation for this dependency, it is considered as implausible.

- known/unknown: A dependency is considered as known if the expert was aware of this dependency prior to the interview. If the expert was not aware of this dependency prior to the interview, it is considered as unknown.

Overall, we discussed 89 feature dependencies in depth.

For RQ 3, each interview transcript was analyzed through a process of coding: breaking up the interviews into smaller coherent units (sentences or paragraphs), and adding codes (representing key characteristics) to these units. For this purpose, we asked the experts for experiences in their work where misconceptions about feature interactions led to errors and increased efforts in the system design. This part of the interview should provide information about the need for an extensive modeling of feature interactions.

\section{Analysis Procedures}

The procedure for the analysis of the functional architecture varies for the three research questions:
For RQ 1, we analyzed the vehicle feature graph in order to assess the ratio of vehicle features that are dependent on another vehicle feature and to count the number of incoming and outgoing dependencies between vehicle features. We also measured the dependency fan-in and fan-out as well as the PageRank [13] for all vehicle features on the vehicle feature graph in order to see whether dependencies are distributed equally or if certain vehicle features are more central than others. Thus, we obtain information about the extent and distribution of functional dependencies in real automotive software systems.

For RQ 2, we counted the number and ratio of dependencies for each combination of category values, leading to a $2 \times 2$ matrix with the two categories as dimensions. We especially investigated the ratio of plausible feature dependencies as an indicator for the validity of our quantitative study and the ratio of known feature dependencies as an indicator for the awareness of feature dependencies in general.

For RQ 3, we developed a coding system with 7 codes structured into 3 categories. We assigned these codes to the units of the interviews. Only codes that appeared in more than one interview were considered for the study results.

\section{E. Validity Procedures}

We analyzed the system under investigation at a final stage of the development process where it was already subject to several architectural reviews and testing procedures. Therefore, errors and misconceptions in the functional architecture can nearly be ruled out. However, our analyses show that vehicle features might differ in the way how they are modeled within the functional architecture. To ensure validity we presented and discussed the results with feature experts at the BMW Group, who assessed the found dependencies concerning their plausibility. The evaluation of RQ 2 and RQ 3 is based on semi-formal interviews with feature experts from the BMW Group. In order to get representative results from the interview partners we selected one expert from each area within the domain of driving dynamics and driver assistance. These areas are: lateral, longitudinal and vertical dynamics as well as driver assistance features. The experts were responsible for a number of $12-46$ features.

\section{STUdy RESUlTs}

In this section the results of the study are presented. They are structured according to the defined research questions.

\section{A. Extent and Distribution of Dependencies (RQ 1)}

Analyzing the vehicle feature graph, we found 1,451 dependencies between the 94 vehicle features. Only 9 out of the 94 vehicle features were completely independent from any other vehicle feature. 81 vehicle features were dependent on another vehicle feature (i.e., had incoming dependencies) and 72 vehicle features had an influence on another vehicle feature (i.e., had outgoing dependencies). Table II summarizes these results. There were 234 different logical signals that caused the dependencies. 
TABLE I

EXTENT OF DEPENDENCIES IN THE VEHICLE FEATURE GRAPH

\begin{tabular}{|l||c|c|}
\hline & Number & Ratio \\
\hline all VFs & 94 & $100 \%$ \\
\hline VFs with incoming dependencies & 81 & $86.2 \%$ \\
\hline VFs with outgoing dependencies & 72 & $76.6 \%$ \\
\hline VFs with incoming and outgoing dependencies & 68 & $72.3 \%$ \\
\hline VFs without dependencies & 9 & $9.6 \%$ \\
\hline
\end{tabular}

TABLE II

DISTRIBUTION OF DEPENDENCIES IN THE VEHICLE FEATURE GRAPH

\begin{tabular}{|c||c|c|c|}
\hline & $\begin{array}{c}\text { Dependencies } \\
\text { (Ingoing) }\end{array}$ & $\begin{array}{c}\text { Dependencies } \\
\text { (Outgoing) }\end{array}$ & PageRank \\
\hline Maximum & 48 & 53 & $5.81 \%$ \\
\hline Median & 3 & 11 & $0.72 \%$ \\
\hline Minimum & 0 & 0 & $0.28 \%$ \\
\hline
\end{tabular}

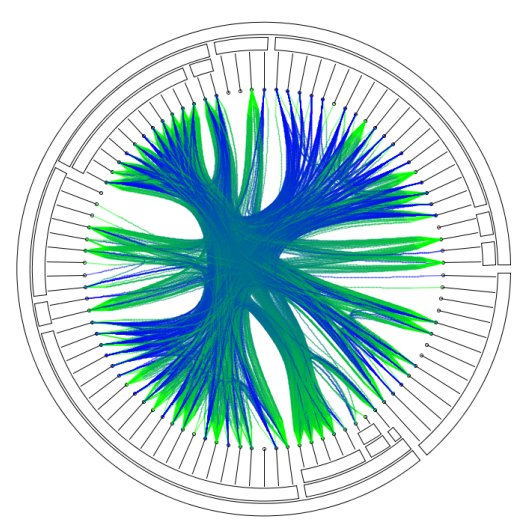

Fig. 5. Vehicle Features and their dependencies visualized as an Edge Bundle View. The outer ring represents the hierarchy of vehicle features. Each dot on the inside of the outer ring is an atomic vehicle feature. The lines indicate a dependency between two features.

The distribution of the dependencies shows that dependencies between vehicle features are distributed all over the system. However, there are some vehicle features that are more central in the sense that they have a large number of dependencies to other vehicle features. Table $[1$ ) shows that a vehicle feature depends on up to 48 other vehicle features, whereas on the other side vehicle features have a maximum of 53 other vehicle features that they influence, which accounts for $56 \%$ of the system features. Most of the features have at least 3 features they depend on and have at least 11 features they influence. The computation of the PageRank [13] gives an idea about the "importance" of single vehicle features and deviates by a factor of almost 20. This intermeshed structure becomes particularly visible when illustrating the dependencies as an Edge Bundle View [14] (see Fig. 5).

\section{B. Awareness of Dependencies (RQ 2)}

Table III summarizes the results of the expert interviews that we conducted in order to assess the plausibility and awareness of the analyzed feature dependencies.
TABLE III

PLAUSIBILITY AND AWARENESS OF THE ANALYZED FEATURE DEPENDENCIES $(\mathrm{N}=100)$

\begin{tabular}{|c||c|c||c|}
\hline & known & unknown & sum \\
\hline \hline plausible & $41.0 \%$ & $48.0 \%$ & $89.0 \%$ \\
\hline implausible & $1.0 \%$ & $10.0 \%$ & $11.0 \%$ \\
\hline \hline sum & $42.0 \%$ & $58.0 \%$ & $100 \%$ \\
\hline
\end{tabular}

The results indicate that our analysis produced reasonable results as only $11 \%$ of the examined feature dependencies were considered as implausible, i.e., the dependencies were a result of our analysis but the experts considered them as not correct or at least they were not able to give account of them. Of the 100 feature dependencies that we examined, $42 \%$ were known to the experts and 58\% were unknown. Most of the feature dependencies that we examined were considered as unknown but plausible, i.e., the experts were not aware of the dependency between the features but when examining the affected signals and leaf functions they found reasonable explanations for them. One examined dependency was considered as known and implausible as the expert were aware of it but had no explanation why this dependency exists.

\section{Importance of Dependencies ( $R Q 3)$}

Our interviews reveal that the knowledge about feature dependencies is especially important for impact analyses on features and signals. The experts for example mentioned: "Feature dependencies are important for the assessment of the complexity, especially when considering the impact of errors" and "It is important to know who uses the signals that features in my responsibility provide". However, the interviews also revealed several problems in the elicitation and revelation of these dependencies. Two main reasons for that are incomplete documentation and dependencies that arise from architectural decisions. The experts said: "Many dependencies arise from specific local signals that are provided by a central leaf function and used by a lot of features" and "Dependencies between features that are known to function correctly together are not explicitly documented". As a major potential benefit of a rigorous documentation of feature dependencies, the experts named the precise tracing of logical signals and architectural decision to requirements. They said: "Tracing links between requirements and architectural decisions would be very useful" and "A back-link from logical signals to the requirements that caused them would be beneficial".

\section{Discussion}

\section{A. Threats to Validity}

A threat to the internal validity is the fact that the analyzed model is already a realization/implementation of the system features. Dependencies might thus be a consequence of a design decision made by a developer and not an integral part of the system features itself. Another threat pertains to the definition of dependency as given in this paper. Besides the explicitly modeled dependencies that are in the focus of 
this paper, there may also be dependencies between vehicle features that occur when features are implicitly connected through a feedback loop through the environment.

A threat to the external validity is that we performed this study in a development and tooling context specific to the Driving Dynamics and Driver Assistance department of the BMW group. This context might not be transferable to other companies or domains. However, from our experience, we are confident that the definition of system features that are implemented by a network of functional blocks is pretty much standard in the development of automotive software systems.

\section{B. Impact / Implications}

The conclusions we draw point at a number of problems that occur in today's development of automotive software systems. Current development processes handle vehicle features more or less as isolated units of functionality [6]. This has to some extent historical reasons as the automotive industry managed to make their different functionality as independent as possible such that vehicles could be developed and produced in a highly modular way. With the coming up of software-based functions in the vehicle this independence disappeared [6].

Furthermore, our results show that developers consider the knowledge about functional dependencies as important, especially for tracing purposes and impact analyses. Architectural decisions hide and scatter these dependencies, which leads to the large number of unknown feature dependencies as reported in the last section. An interesting point is that the reasons for feature dependencies that were considered as implausible can also be related to architectural concerns. Leaf functions are architectural elements, which are subject to reuse and thus related to a number of features. Developers use leaf functions without considering other vehicle features that might also affect or be affected by this leaf function. The emerging feature dependencies were, in most cases, considered as implausible.

Therefore, we argue that these dependencies need to be modeled precisely on the level of vehicle features, still independent from any architectural design decisions (cf. [15]).

\section{Relation to Existing Evidence}

The results of RQ 1 reflect the results of a study we have performed with another automotive company, in which we analyzed the software architecture of a truck [9]. In that study, vehicle features showed a comparable extent of dependencies, i.e., at least $69 \%$ of the analyzed vehicle features depend on other vehicle features or influence other vehicle features. The analyzed software system of that study was smaller and contained only 55 vehicle features.

Our results back up the challenges mentioned in [16] and [6], where the authors state that features do not stand alone, but exhibit a high dependency on each other, so that a vehicle becomes a complex system where all functions act together.

\section{CONClusions And Future WORK}

The results of this paper show that dependencies between vehicle features pose a great challenge for the development of automotive software systems. Not only that almost every vehicle feature depends on and/or influences another vehicle feature, we have also seen that modeling the dependencies on an architectural level is insufficient for analyzing them, leading to a $50 \%$ chance that a developer is not aware of a specific dependency. In our study this was particularly striking when the feature dependencies arose from architectural decisions. Considering these conclusions we plan to further discuss our results with the developers in order to integrate the modeling of dependencies on the level of vehicle features. Therefore, we have to specify features more precisely, for example by annotating them with inputs and outputs, and define the dependencies based on this notion of a vehicle feature (cf. [15]). We especially plan to integrate features into a feature hierarchy and describe the dependencies between features by means of a mode concept [17]. This structured specification models dependencies independently from architectural decisions and thus facilitates the modeling of feature interactions in requirements engineering.

\section{REFERENCES}

[1] M. Jackson and P. Zave, "Distributed Feature Composition: A Virtual Architecture for Telecommunications Services," IEEE Trans. Software Eng, vol. 24, no. 10, 1998.

[2] K. Kang, S. Cohen, J. Hess, W. Novak, and S. Peterson, "FeatureOriented Domain Analysis (FODA) Feasibility Study," Software Engineering Institute, Carnegie Mellon University, Tech. Rep., 1990.

[3] B. Schätz, "Modular functional descriptions," Electronic Notes in Theoretical Computer Science, vol. 215, 2008.

[4] P. Zave, "Requirements for Evolving Systems: A Telecommunications Perspective," in 5th IEEE International Symposium on Requirements Engineering. IEEE Computer Society, 2001.

[5] S. Benz, "Generating Tests for Feature Interaction," Ph.D. dissertation, Technische Universität München, 2010.

[6] M. Broy, "Challenges in automotive software engineering," in Proceedings of the 28th international conference on Software engineering. New York, NY, USA: ACM, 2006.

[7] M. Broy, I. Krüger, A. Pretschner, and C. Salzmann, "Engineering Automotive Software," Proceedings of the IEEE, vol. 95, no. 2, 2007.

[8] C. Heitmeyer, "Using the SCR* Toolset to Specify Software Requirements," Industrial-Strength Formal Specification Techniques, 1998.

[9] A. Vogelsang, S. Teuchert, and J. Girard, "Extent and characteristics of dependencies between vehicle functions in automotive software systems," in Modeling in Software Engineering (MISE), 2012 ICSE Workshop on, 2012.

[10] M. Calder and E. H. Magill, Eds., Feature Interactions in Telecommunications and Software Systems VI. IOS Press, 2000.

[11] M. Fowler and K. Scott, UML distilled - a brief guide to the Standard Object Modeling Language (2. ed.). Addison-Wesley-Longman, 2000.

[12] C. L. Heitmeyer, J. Kirby, and B. G. Labaw, "The scr method for formally specifying, verifying, and validating requirements: Tool support," in ICSE, 1997.

[13] S. Brin and L. Page, "The anatomy of a large-scale hypertextual web search engine," Computer Networks and ISDN Systems, vol. 30, 1998, proceedings of the Seventh International World Wide Web Conference.

[14] D. Holten, "Hierarchical edge bundles: Visualization of adjacency relations in hierarchical data," Visualization and Computer Graphics, IEEE Transactions on, vol. 12, no. 5, 2006.

[15] M. Broy, "Multifunctional software systems: Structured modeling and specification of functional requirements," Science of Computer Programming, vol. 75, no. 12, 2010.

[16] A. Pretschner, M. Broy, I. H. Krüger, and T. Stauner, "Software Engineering for Automotive Systems: A Roadmap," in 2007 Future of Software Engineering. IEEE Computer Society, 2007.

[17] M. Broy, W. Damm, S. Henkler, K. Pohl, A. Vogelsang, and T. Weyer, "Introduction to the SPES Modeling Framework," in Model-Based Engineering of Embedded Systems. Springer Berlin Heidelberg, 2012. 